\title{
NOTAS CIENTÍFICAS \\ Influência da minhoca Pontoscolex corethrurus na distribuição do acaricida dicofol em um Argissolo(1)
}

\begin{abstract}
Tomaz Langenbach(2), Márcia Verônica de Souza Inácio(3), Adriana Maria de Aquino ${ }^{(4)}$ e Beate Brunninger ${ }^{(5)}$

Resumo - O objetivo deste trabalho foi estudar a importância da minhoca Pontoscolex corethrurus na distribuição do pesticida dicofol em um Argissolo. Como modelo foram utilizados microcosmos contendo solo tamizado e acondicionado na densidade $1,25 \mathrm{~g} \mathrm{~cm}^{-3}$. Em microcosmos com e sem Pontoscolex corethurus, foram aplicados ${ }^{14} \mathrm{C}$-dicofol, e após um período de 52 dias fez-se uma simulação de chuvas torrenciais. Na camada de $0-1 \mathrm{~cm}$, recuperou-se $75 \%$ da radioatividade no solo sem minhoca, e no solo com minhoca, a recuperação foi $9 \%$ inferior. Nas camadas mais profundas, os valores da radioatividade ficaram abaixo de $20 \%$, e as diferenças entre os tratamentos não ultrapassaram 2\%. Esta espécie de minhoca, muito freqüente no Brasil, mostrou não ter influência relevante na distribuição do pesticida no solo.
\end{abstract}

Termos para indexação: pesticida, organoclorados, fauna do solo, radioatividade, transporte no solo, poluição do solo.

\section{Effects of earthworm Pontoscolex corethrurus on distribution of acaricida dicofol in a Podzolic soil}

\begin{abstract}
The aim of this work was to study the influence of earthworm on pesticides distribution in a Podzolic soil. The experimental model used was a microcosm filled with sieved soil to a final density of $1.25 \mathrm{~g} \mathrm{~cm}^{-3}$. In microcosms with or without Pontoscolex corethurus ${ }^{14} \mathrm{C}$-dicofol was applied, and after a period of 52 days strong rain simulation was performed. In the layer of $0-1 \mathrm{~cm} 75 \%$ of the radioactivity in the soil without earthworms were recovered, and in the soil with earthworms the recovery was $9 \%$ inferior. In the deepest layers the values of the radioactivity were below $20 \%$ and the differences among the treatments did not surpass $2 \%$. This earthworm species of large occurrence in Brazil showed no important influence on the distribution of the pesticide in soil.

Index terms: pesticides, organochlorides, soil fauna, radioactivity, soil transport processes, soil pollution.
\end{abstract}

\footnotetext{
(1) Aceito para publicação em 23 de maio de 2002.

(2) Universidade Federal do Rio de Janeiro (UFRJ), Instituto de Microbiologia, Centro de Ciências da Saúde, Cidade Universitária, Bloco I, Ilha do Fundão, CEP 21941-590 Rio de Janeiro, RJ. E-mail: langenbach@micro.ufrj.br

(3) Rua Dr. João Maria 91, casa 34, Parque João e Maria, CEP 28024-600 Campos dos Goytacazes, RJ.

(4) Embrapa-Centro Nacional de Pesquisa de Agrobiologia, Km 47, antiga Rod. Rio-São Paulo, CEP 23850-970 Seropédica, RJ. E-mail: adriana@cnpab.embrapa.br

(5) Gänshaller Strasse 32, 94099 Schmidham/Ruhstorf a.d. Rott, Alemanha.
} 
Por serem suscetíveis à lixiviação no solo, os agrotóxicos podem atingir as águas subterrâneas. Essas águas não só podem ser utilizadas em poços para consumo doméstico, como podem atingir rios, e, conseqüentemente, entrar na cadeia alimentar e afetar populações de animais em todos os níveis (Gliessman, 2000). Neste aspecto, é muito importante gerar conhecimentos para o entendimento dos fatores que influenciam o maior ou menor deslocamento dos agrotóxicos no solo.

As práticas agrícolas como o plantio direto promovem maior acúmulo de matéria orgânica e pouca perturbação do solo, criando, deste modo, condições para o estabelecimento das minhocas. Edwards et al. (1993) demonstraram que nessas situações as minhocas influenciaram a distribuição dos agrotóxicos no solo e na lixiviação de agroquímicos, principalmente em épocas de alta pluviosidade. Vários estudos têm demonstrado que pela ação das minhocas podem formar os macroporos (Beven \& Germann, 1982; Pivetz \& Steenhuis, 1995) e galerias orientadas verticalmente (Zachaman et al., 1987). Dessa forma, os macroporos e as galerias promovem a ocorrência do fluxo preferencial de solutos, conduzindo os agroquímicos para horizontes mais profundos do solo (Sadeghi \& Isensee, 1994).

A maioria dos trabalhos que abordam essa questão foi realizada em regiões de clima temperado e refere-se às espécies de minhocas como Lumbricus terrestris, por exemplo, que não são amplamente encontradas no Brasil. Esse grupo se alimenta predominantemente da matéria orgânica que está na superfície do solo. Após a ingestão, esses materiais passam através do trato digestivo e são depositados como coprólitos nos horizontes mais profundos do solo (Lee, 1985). Os efeitos disso sobre as propriedades físicas, químicas e biológicas do solo influenciam a persistência, a biodisponibilidade e o transporte de agrotóxicos no solo (Stehouwer et al., 1994; Pivetz \& Steenhuis, 1995; Farenhorst et al., 2000).

Já em agroecossistemas tropicais têm sido verificada a dominância da espécie Pontoscolex corethrurus (Muller) (Aquino, 1999; Aquino et al., 1999). As minhocas dessa espécie regulam muitos processos, visto que ingerem quantidade considerável de solo, cerca de 200-400 t ha ${ }^{-1}$ ano $^{-1}$ (Lavelle et al., 1983). Entretanto, pouco se conhece sobre a influência que essa espécie de minhoca pode exercer na distribuição dos agrotóxicos no solo.

O objetivo do trabalho foi estudar, em condições controladas e sob simulação de chuvas torrenciais, a distribuição do acaricida organoclorado Kelthane, com o princípio ativo ${ }^{14} \mathrm{C}$-dicofol, na presença da Pontoscolex corethrurus (Muller), uma espécie de minhoca com disseminação pan-tropical, muito difundida nos meios antropogênicos.

Foram utilizadas amostras de solo classificado como Argissolo, coletadas no Município de Seropédica, RJ. A análise granulométrica revelou a seguinte composição: $59 \%$ de areia, $12 \%$ de silte e $29 \%$ de argila, e a densidade foi de $1,25 \mathrm{~g} \mathrm{~cm}^{-3}$. As amostras de solo foram destorroadas, secadas à temperatura ambiente e tamisadas em peneira de malha de $2 \mathrm{~mm}$. Em seguida, foram acondicionadas em microcosmos de acrílico com diâmetro de $14,5 \mathrm{~cm}$ e altura de $24,9 \mathrm{~cm}$. Os microcosmos foram forrados com papel de alumínio. Na parte inferior desses microcosmos, foi colocada uma tela para reter o solo. 
Os tratamentos foram com e sem minhocas, com três repetições, totalizando seis microcosmos. Foram introduzidas cinco minhocas adultas da espécie Pontoscolex corethurus Muller. Todos os microcosmos foram cobertos com cerca de $50 \mathrm{~g}$ (peso fresco) de grama-batatais como fonte de alimento para as minhocas, renovados a cada semana. Os microcosmos foram irrigados de modo a atingir $40 \%$ da capacidade máxima de retenção de água. A manutenção da umidade foi feita pela correção de peso. A atividade das minhocas foi acompanhada pela observação visual da presença de coprólitos na superfície dos microcosmos. Foi realizada análise dos macro e micronutrientes do solo com e sem minhocas, de acordo com o método da Embrapa (1979).

Após 62 dias da instalação do experimento, aplicaram-se, por microcosmo, $2 \mathrm{~mL}$ da solução de dicofol (Riedel). Essa solução foi preparada contendo 2,02 $\mathrm{mg} \mathrm{mL}^{-1}$ (solvente $2 \mathrm{~mL}$ acetona: $10 \mathrm{~mL} \mathrm{H}_{2} \mathrm{O}$ ), ao qual se adicionaram 17.100 Bq de ${ }^{14} \mathrm{C}$-dicofol obtido da Rohm e Haas Co., com atividade específica de 7,4 mCi mmol ${ }^{-1}$ e com marcação universal de carbono.

Após 52 dias da aplicação do ${ }^{14} \mathrm{C}$-dicofol, simularam-se chuvas intensas de $200 \mathrm{~mL}$ com água destilada, em intervalos de uma hora, correspondendo a um total de $122 \mathrm{~mm}$ em 10 horas. Em seguida, o solo foi retirado dos microcosmos, fracionado em camadas de $1 \mathrm{~cm}$ até a quinta fração, a partir da qual o fracionamento foi de $5 \mathrm{em} 5 \mathrm{~cm}$. As amostras de solo em torno de 1 a $2 \mathrm{~mm}$ da galeria das minhocas (drilosfera) foram removidas, respeitandose as respectivas camadas. Para determinação da radioatividade, as frações de solo foram secadas à temperatura ambiente, trituradas, pesadas e incineradas num Oxidizer OX-500, o qual oxida toda a matéria orgânica a $\mathrm{CO}_{2}$, que por sua vez é adsorvido por solução cintiladora (aguasolv: 4 metanol: 2,5 etanolamina). As minhocas e os casulos também foram preparados conforme o procedimento descrito acima. A radioatividade das amostras de solo, casulos e minhocas, foi quantificada num cintilador líquido da Beckman. Para avaliar a radioatividade no lixiviado, foram colocados $5 \mathrm{~mL}$ da alíquota em $5 \mathrm{~mL}$ da solução cintiladora aquasolv ( $4 \mathrm{~g}$ de PPO, 0,25 g de POPOP, $333 \mathrm{~mL}$ de triton $\mathrm{x}-100$ e $667 \mathrm{~mL}$ de tolueno) e medido no cintilador.

Para determinação do ${ }^{14} \mathrm{C}$-dicofol extraível, foram tomados $10 \mathrm{~g}$ de solo seco e triturado, de cada camada do solo do microcosmo com e sem minhoca, e adicionaram-se $200 \mathrm{~mL}$ de hexano. Após 40 minutos de incubação num agitador, as amostras foram submetidas por 20 segundos a banho ultra-som. As amostras foram centrifugadas a $4.300 \mathrm{~g}$ durante 10 minutos, e o sobrenadante, recolhido e colocado em frascos de cintilação líquida até completa secura. A amostra de solo foi tratada mais duas vezes com $10 \mathrm{~mL}$ de hexano, e o sobrenadante, adicionado aos frascos de cintilação líquida até a secura completa. Após a secura do último sobrenadante, adicionaram-se $10 \mathrm{~mL}$ de solução cintiladora e mediram-se as amostras no cintilador Beckman.

Durante todo o experimento foi observada a produção de coprólitos na superfície do solo dos microcosmos, o que indica que as minhocas sobreviveram na concentração máxima de $11,4 \mathrm{mg} \mathrm{kg}^{-1}$ de dicofol na camada superficial de $1 \mathrm{~cm}$.

A análise de solo revelou que os macroelementos $\mathrm{P}$ e $\mathrm{K}$ apresentaram maiores valores nas amostras de solo com minhocas, comparado aos do solo sem minhocas. Concentrações bastante variadas foram observadas em 
micronutrientes na presença de minhocas, alcançando valores aproximadamente cinco vezes maior, como no caso do Fe (Tabela 1). A presença de P. corethrurus influenciou também o $\mathrm{pH}$ do solo, que subiu de $\mathrm{pH}$ 5,0 para 5,8. Os maiores teores de $\mathrm{P} \mathrm{e} \mathrm{K}$, assim como micronutrientes, indicam que as minhocas promovem aumento da fertilidade do solo, como já observado por Lee (1985). Considerando que os métodos de análise utilizados simulam a absorção desses nutrientes pela planta, o efeito observado demonstra que a minhoca favorece a disponibilidade destes elementos para a planta.

As minhocas incorporaram ${ }^{14} \mathrm{C}$-dicofol na ordem de $2,5 \mathrm{dpm} \mathrm{mg}^{-1} \mathrm{em} \mathrm{seu}$ corpo, semelhantes às concentrações contidas no solo, e portanto, não houve bioacumulação, conforme observado também por Belfroid \& Berg (1995). Foi detectada radioatividade no casulo, com valores inferiores a $0,3 \mathrm{dpm} \mathrm{mg}^{-1}$. Os valores contidos no solo em torno das galerias das minhocas (drilosfera) acompanhou a distribuição da radioatividade no solo adjacente, sendo no primeiro centímetro superficial igual a $2,9 \mathrm{dpm} \mathrm{g}^{-1}$, e na última camada, igual a $0,02 \mathrm{dpm} \mathrm{mg}^{-1}$, sem apresentar acumulação.

Foram recuperados na camada de $0-1 \mathrm{~cm}, 75 \%$ da radioatividade no solo sem minhoca, e no solo com minhoca, a recuperação foi $9 \%$ inferior. Nas camadas mais profundas, os valores da radioatividade ficaram abaixo de $20 \%$, e as diferenças entre os tratamentos não ultrapassaram 2\% (Figura 1).

Tabela 1. Análise química de solo com e sem minhocas, após incubação com o acaricida Kelthane organoclorado, cujo princípio ativo é o dicofol marcado.

\begin{tabular}{|c|c|c|c|c|c|c|c|c|c|c|c|c|c|}
\hline Tratamento & $\mathrm{pH}$ & $\mathrm{P}$ & $\mathrm{K}$ & $\mathrm{Ca}$ & $\mathrm{Mg}$ & $\mathrm{Al}$ & $\mathrm{H}$ & $\mathrm{Na}$ & $\mathrm{C}$ & $\mathrm{Fe}$ & $\mathrm{Cu}$ & $\mathrm{Zn}$ & $\mathrm{Mn}$ \\
\hline Sem minhoca & 5,0 & 0,0003 & 0,0176 & 3,3 & 2,2 & 0,1 & 4,1 & 0,03 & 1,79 & 42 & 0,4 & 8,5 & 56,6 \\
\hline Com minhoca & 5,8 & 0,0006 & 0,0306 & 4,1 & 2,2 & 0,1 & 2,4 & 0,08 & 2,1 & 237 & 1,6 & 14,2 & 211,2 \\
\hline
\end{tabular}

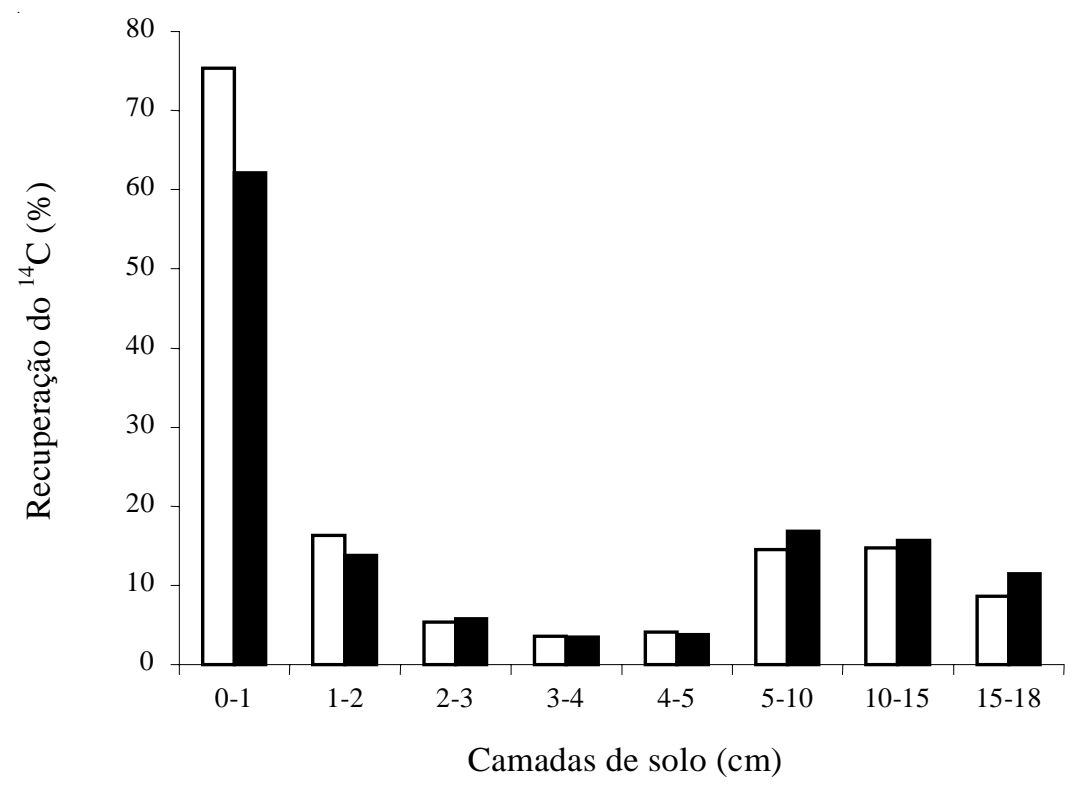

Figura 1. Distribuição do dicofol em diferentes camadas de solo expressa pela porcen-

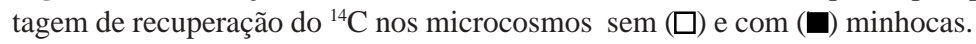


Isto mostra que a distribuição decorrente do movimento do solo pelas minhocas assim como o maior deslocamento da água, decorrente da simulação de chuvas torrenciais, não foi fator determinante da distribuição do pesticida no solo, nas condições estudadas. O total recuperado da radioatividade em todas as frações do solo foi próximo a $100 \%$, e a baixa recuperação no lixiviado $(0,1 \%)$ em oito horas, com chuvas simuladas, num total de $13,5 \mathrm{~mm}$, indicam uma adsorção preferencial do dicofol ao solo. Independentemente da presença ou não das minhocas, o tratamento do solo com hexano somente extraiu $6 \%$ de dicofol radioativo. O hexano é um forte extrator de uma molécula lipofílica como o dicofol; esse resultado indica que há uma ligação forte desse produto ao solo. Dessa forma, entende-se que esse produto resiste às forças de convecção. Apesar disso, não pode ser excluída a possibilidade de uma alteração nessa distribuição, caso ocorra alta pluviosidade pouco tempo após a aplicação do pesticida, que em grande parte pode ainda não estar adsorvido.

A permanência prolongada da $P$. corethrurus no solo em presença do dicofol antes da simulação de chuva permite inferir que também não houve redistribuição deste acaricida pela movimentação mecânica do solo por ação das minhocas. Este dado é compatível com a radioatividade encontrada na drilosfera em relação ao solo, diferente das observações de Czapar et al. (1992), Edwards et al. (1992) e Stehouwer et al. (1993, 1994). Esses autores observaram que as alterações nas características do solo na drilosfera de Lumbricus terrestris afetaram a adsorção e o transporte de atrazina. A redistribuição de pesticidas no solo pelas minhocas pode ser atribuída a sua estratégia ecológica e em particular aos hábitos alimentares. A espécie $P$. corethrurus assimila eficientemente a matéria orgânica do solo, enquanto $L$. terrestris se alimenta da matéria orgânica da superfície do solo. Assim, esta última constrói galerias subterrâneas predominantemente verticais, enquanto as outras constroem galerias predominantemente horizontais e com um processo de alimentação semelhante à passagem de um tubo flexível, mantendo o solo na mesma posição. Esta lógica sugere que o $P$. corethrurus não redistribuiria outros pesticidas no solo, assim como é possível que outras espécies de minhocas que tenham o mesmo hábito não redistribuam os pesticidas.

Os resultados obtidos mostram que nas condições utilizadas não há alteração na distribuição do dicofol pela ação da $P$. corethrurus no solo. Não há acumulação do dicofol nas margens dos canais (drilosfera) no solo e nem no corpo de $P$. corethrurus.

\section{Agradecimentos}

A Edir Martins Ferreira, pelo auxílio técnico; ao CNPq/DLR (Cooperação com Alemanha), FUJB e à Faperj, pelo apoio financeiro.

\section{Referências}

AQUINO, A. M. de. Meso e macrofauna do solo e sustentabilidade agrícola: perspectivas e desafios para o século. In: CONGRESSO BRASILEIRO DE CIÊNCIA DO SOLO, 27., 1999, Brasília. Anais... Brasília: Sociedade Brasileira de Ciência do Solo, 1999. Palestra 504b. CD-ROM.

AQUINO, A. M. de; SILVA, E. M. R. da; PEREIRA, J. C.; RICCI, M. dos S. F.; REIS, V. M. Transformações biológicas e microbiológicas ocorridas no solo de 
um cafezal convencional em conversão para orgânico. Seropédica: EmbrapaCNPAB, 1999. 10 p. (Comunicado Técnico, 31).

BELFROID, A.; BERG, M. van der. Uptake, bioavailability and elimination of hydrophobic compounds in earthworms (Eisenia andrei) in field-contaminated soil. Environmental Toxicology and Chemistry, New York, v. 14, n. 2, p. 605-612, 1995.

BEVEN, K.; GERMANN, P. F. Macropores and water flow in soils. Water Resources Research, Washington, v. 18, p. 1311-1325, 1982.

CZAPAR, G. F.; HORTON, R.; FAWCETT, R. S. Herbicide and tracer movement in soil columns containing an artificial macropore. Journal of Environmental Quality, Madison, v. 21, n. 1, p. 110-115, 1992.

EDWARDS, W. M.; SHIPITALO, M. J.; OWENS, L. B.; DICK, W. A. Factors affecting preferential flow of water and atrazine through earthworm burrows under continuous no-till corn. Journal of Environmental Quality, Madison, v. 22, n. 3, p. 453-457, 1993.

EDWARDS, W. M.; SHIPITALO, W. A.; DICK, W. A.; OWENS, L. B. Rainfall intensity affects transport of water and chemicals through macropores in no-till soil. Soil Science Society of America Journal, Madison, v. 56, p. 52-58, 1992.

EMBRAPA. Serviço Nacional de Levantamento e Conservação de Solos (Rio de Janeiro, RJ). Manual de métodos de análise de solo. Rio de Janeiro, 1979. Não paginado.

FARENHORST, A.; TOPP, E.; BOWMAN, B. T.; TOMLIN, A. D. Earthworms and the dissipation and distribution of atrazine in soil profile. Soil Biology and Biochemistry, Amsterdam, v. 32, p. 23-33, 2000.

GLIESSMAN, S. R. Agroecologia: processos ecológicos em agricultura sustentável. Porto Alegre: Editora da UFRGS, 2000. 653 p.

LAVELLE, P.; RANGEL, P.; KANYONYO, J. Mucus production by two species of tropical earthworms: Millsonia lamtoiana (Megascolecidae) and Pontoscolex corethrurus (Glossoscolecidae). In: LEBRUM, P.; ANDRÉ, H.; DEMEDTS, A.; GREGOIRE-WIBO, C.; WAUTHY, G. (Ed.). New trends in soil biology. Louvainla-Neuve: Dieu-Brichard, 1983. p. 405-410.

LEE, K. E. Earthworms: their ecology and relationships with soils and land use. London: Academic, 1985.411 p.

PIVETZ, B. E.; STEENHUIS, T. S. Soil matrix and macropore biodegradation of 2,4-D. Journal of Environmental Quality, Madison, v. 24, n. 4, p. 564-570, July/Aug. 1995.

SADEGHI, A. M.; ISENSEE, A. R. Spatial distribution of atrazine residues in soil and shallow groundwater: effect. Agriculture, Ecosystems and Environment, Amsterdam, v. 48, n. 1, p. 67-76, 1994.

STEHOUWER, R. C.; DICK, W. A.; TRAINA, S. J. Characteristics of earthworm burrow lining affecting atrazine sorption. Journal of Environmental Quality, Madison, v. 22, n. 1, p. 181-185, 1993.

STEHOUWER, R. C.; DICK, W. A.; TRAINA, S. J. Sorption and retention of herbicides in vertically earthworm and artificial burrows. Journal of Environmental Quality, Madison, v. 23, n. 2, p. 286-292, 1994.

ZACHAMAN, J. E.; LINDEN, D. R.; CLAPP, C. E. Macroporous infiltration and redistribution as affected by earthworms, tillage and residue. Soil Science Society of America Journal, Madison, v. 51, p. 1580-1586, 1987. 